\title{
Perspectivas eTwinning en la educación superior: boceto del esqueleto curricular
}

\section{Amparo Bonet Juan ${ }^{a}$, Jordi Capó Vicedo ${ }^{b}$ y Antonio Giménez-Morera ${ }^{c}$}

a Universitat Politècnica de València, Department of Economy and Social Sciences, Camino de Vera s/n, 46022, Valencia, Spain, ambojua@doctor.upv.es, ${ }^{b}$ Centre for Research in Business Management (CEGEA), Universitat Politècnica de València, Spain. jorcavi0@esp.upv.es. ${ }^{c}$ Universitat Politècnica de València, Department of Economy and Social Sciences, Camino de Vera s/n, 46022, Valencia, Spain angimo1@doctor.upv.es.

\begin{abstract}
The challenge of an eTwinning project is to achieve learning through collaboration and innovation between different European centers. This paper represents a collaborative work aimed at higher education students. It is a comparative study and the repercussion about the employability and the job opportunities of our students. The study concludes with a proposal for collaborative learning through an eTwinning project.
\end{abstract}

Keywords: eTwinning, educational innovation, collaborative learning, information and communication technology, higher education.

\begin{abstract}
Resumen
El reto de un proyecto eTwinning es lograr el aprendizaje mediante la colaboración y la innovación entre diferentes centros europeos. El presente artículo representa la materialización de un trabajo de colaboración dirigido a alumnos de educación superior. Se trata de un estudio comparativo entre el comercio y las acciones de Marketing llevadas a cabo por las empresas en función del país en el que se encuentran y la repercusión que ello tiene, en la empleabilidad y las oportunidades laborales de nuestros alumnos a nivel europeo. El estudio concluye con una propuesta curricular para el aprendizaje colaborativo a través de un proyecto eTwinning.
\end{abstract}

Palabras clave: eTwinning, innovación educativa, aprendizaje colaborativo, tecnologías de la información y la comunicación, educación superior.

\section{Introducción}

eTwinning forma parte de Erasmus+, el programa de la Unión Europea en materia de educación, formación, juventud y deporte. eTwinning está organizado a nivel europeo por el Servicio Central de Apoyo (SCA), con sede en Bruselas (http://www.etwinning.net) y a nivel nacional está coordinado por los respectivos Servicios Nacionales de Apoyo. En España, el Servicio Nacional de Apoyo (SNA) pertenece al Instituto Nacional de Tecnologías Educativas y de Formación del Profesorado (INTEF) del Ministerio de 
Educación, Cultura y Deporte, desde donde trabaja en coordinación con todas las Comunidades y Ciudades Autónomas.

$\mathrm{Su}$ objetivo principal es promover el contacto y la colaboración entre centros, ya sea de un mismo país o de distintos países europeos. Facilitar un entorno para el desarrollo profesional docente, el intercambio de ideas y para establecer proyectos educativos sobre cualquier tema del ámbito escolar, basados en el trabajo en colaboración, el desarrollo de las competencias clave, el uso de las TIC y la potenciación de la dimensión europea.

Hay que destacar los aspectos positivos de estos hermanamientos: el sentido de creación de comunidad con otros centros; las posibilidades de aprendizaje entre pares, ya sea entre docentes o entre alumnos; pero, sobre todo, la posibilidad de compartir ideas y experiencias para el crecimiento personal o colectivo dentro de la sociedad europea. Los participantes dentro de niveles educativos no universitarios, también conocidos como eTwinners, indican una mejora significativa tanto en la motivación como en el rendimiento académico (Galvin, 2009). Los alumnos, encuentran motivador el trabajo y el contacto con compañeros de otros países. El hecho de implementar un nuevo tipo de metodología, buscando la colaboración con otros agentes (otro centro educativo, otros docentes, otros alumnos,...), la inclusión de otras realidades, etc. hacen que los alumnos muestren un interés especial por el aprendizaje y el posterior éxito.

En consecuencia, fomenta el sentido de equipo, donde también los docentes experimentan metodologías diferentes y buscan sinergias que produzcan cambios en las aulas y centros educativos. Además, se ofrecen oportunidades de desarrollo profesional en un entorno seguro, donde el trabajo realizado tiene reconocimiento con Sellos de calidad o premios tanto nacionales como Europeos.

\section{Antecedentes}

La presencia eTwinning en la educación superior es escasa. Ello se debe a las características tanto de los estudiantes como de los docentes que dificultan la puesta en marcha de un proyecto de estas características. Partiendo de esta premisa inicial, en este artículo se presenta el boceto curricular de un proyecto interdisciplinar de colaboración europea para la concienciación de nuestros alumnos hacia las oportunidades laborales en el mercado europeo. Alianzas estratégicas para la educación y formación profesional mediante el intercambio de prácticas profesionales.s[íp]

\subsection{Datos generales}

La siguiente tabla, presenta en los datos descriptivos del proyecto eTwinning que lleva por título "Market inspiration".

Tabla 1. Datos descriptivos del Proyecto eTwinning

\begin{tabular}{ll}
\hline Título & "Market inspiration” \\
\hline Tema & Comercio, Marketing y mercado laboral europeo. \\
Descripción & Se trata de un estudio comparativo entre el comercio y las acciones de \\
\hline
\end{tabular}


Marketing llevadas a cabo por las empresas en función del país en el que se encuentran y la repercusión que ello tiene, en la empleabilidad y las oportunidades laborales de nuestros alumnos a nivel europeo.

\begin{tabular}{|c|c|}
\hline Edad & 18 años de edad en adelante \\
\hline Número de alumnos & 25 alumnos aprox. \\
\hline Etapa & Formación Profesional superior \\
\hline Áreas educativas & 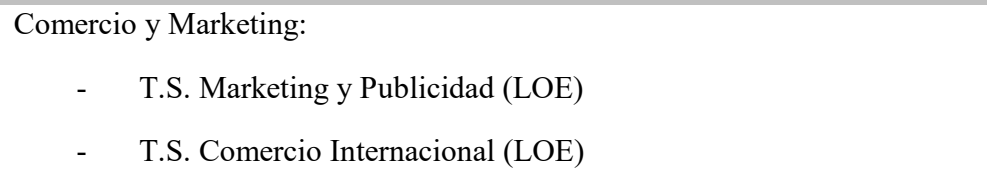 \\
\hline Módulos & $\begin{array}{ll}\text { - } & \text { Formación y Orientación Laboral. } \\
\text { - } & \text { Inglés Técnico } \\
\text { - } & \text { Políticas de Marketing (1GS Marketing y Publicidad) } \\
\text { - } & \text { Trabajo de campo en la investigación comercial (2GS Marketing y } \\
& \text { Publicidad) } \\
\text { - } & \text { Marketing internacional (2GS Comercio Internacional) }\end{array}$ \\
\hline Duración estimada & Curso escolar completo \\
\hline Socios & España y Portugal \\
\hline Competencias básicas & $\begin{array}{l}\text { Idiomas, digitales, aprender a aprender, sociales y cívicas, iniciativa y } \\
\text { emprendimiento y expresión cultural }\end{array}$ \\
\hline
\end{tabular}

Fuente: Elaboración propia

\subsection{Objetivos}

\subsubsection{Objetivos genéricos}

Se trata de un proyecto interdisciplinar de colaboración europea para la concienciación de nuestros alumnos hacia las oportunidades laborales en el mercado europeo. Alianzas estratégicas para la educación y formación profesional mediante el intercambio de prácticas profesionales.

En términos genéricos se trata de:

1. Promover la comunicación y la colaboración entre alumnos y empresas de diferentes países europeos.

2. Ampliar su entorno cultural y compartir sus conocimientos con los demás.

3. Identificar y describir las similitudes y diferencias de los comercios de los diferentes países participantes, las acciones de marketing que llevan a cabo y las posibilidades de entrar en su mercado laboral.

4. Ser conscientes del mundo globalizado. 
5. Mejorar las habilidades comunicativas en otra lengua.

6. Uso de las TIC para comunicarse eficazmente.

7. Aumentar la motivación del alumnado en el aprendizaje.

8. Incentivar el espíritu emprendedor del alumno.

9. Que al finalizar el proyecto, el alumno visualice la utilidad de los estudios que está realizando.

\subsubsection{Objetivos especificos}

1. Aumentar los conocimientos acerca de los usos y costumbres comerciales y acciones de marketing que desarrollan en el ámbito empresarial en los distintos países europeos.

2. Investigar el tejido empresarial de los países miembros de este proyecto.

3. Análisis de las ayudas que brindan los entes públicos y privados a los jóvenes emprendedores.

4. Aumentar la empleabilidad y las perspectivas laborales de nuestros alumnos de Formación Profesional de Comercio a nivel europeo.

5. Luchar contra el desempleo juvenil en España animando a los alumnos a la investigación del sector comercio, como una de las áreas con mayor desempleo juvenil.

6. Búsqueda activa de empleo en sectores emergentes: empresas de Marketing y publicidad del país vecino: Portugal. Para ello, se ha contactado con un posible socio en Lisboa, cuyo objetivo es mejorar el conocimiento de los distintos mercados y la conciencia cultural de nuestros alumnos. Posible alianza con la empresa: http://www.leoburnett.es/

7. Este proyecto pretende unir sinergias de empresas, escuelas y otras organizaciones.

8. Fomentar en nosotros el intercambio de conocimientos teóricos y experiencias sobre el know how de las empresas extranjeras. Así mismo, se pretende consolidar vínculos entre ambas entidades con la finalidad de crear nuevas relaciones colaborativas (centro integrado de formación profesional-empresa portuguesa).

\subsection{Metodología}

Siguiendo la idea de alianzas europeas para apoyar programas en el sector económico específico se pretende alcanzar las habilidades necesarias para el trabajo en los campos profesionales de los ciclos formativos. Los socios tienen que ofrecer diferentes tipos de negocios. Con lo cual, nuestros alumnos podrán aprender sobre las diferentes conductas y principios comerciales. Queremos acercarnos a las empresas para saber cuáles son las habilidades más valoradas. 


\section{Proceso de trabajo}

Para el inicio del proceso de trabajo, se parte de la elaboración de un estudio comparativo entre el comercio y las acciones de Marketing de las empresas y países participantes. Todo ello, mediante la realización de actividades cuidadas dónde se observa la puesta en común y el trabajo en equipo. Se trata de proporcionar a los estudiantes las habilidades y los medios en su camino hacia el mercado laboral europeo.

\subsection{Fases}

\subsubsection{Fase preliminar: establecer contacto con socios}

Para poder llevar a cabo las alianzas estratégicas, es necesario contar con la colaboración de empresas, centros educativos y organismos públicos.

Para una primera toma de contacto con la realidad profesional, se trabajarán los vínculos para una futura colaboración en proyectos Erasmus y eTwinning en una consultora internacional de Marketing. Se pretende fomentar la posibilidad para nuestros alumnos de prácticas en empresas de la Unión Europea.

Se llevará a cabo un jobshadowing en Portugal (Lisboa) en la empresa http://www.leoburnett.es/, cuyo objetivo es mejorar el conocimiento de los distintos mercados y la conciencia cultural de nuestros alumnos.

En este encuentro, se llevarán a cabo las siguientes tareas:

1. Acordar objetivos, metodología y actividades del proyecto.

2. Acordar la temporalización del trabajo en común.

3. Creación de grupos de trabajo.

4. Acordar alianzas estratégicas para la educación y formación profesional

5. Posibilidad de realizar un período de observación en una empresa internacional consultora de Marketing en Lisboa (Portugal) con el objeto de ampliar las posibilidades de desarrollo profesional del alumnado y profesorado del Ciclo de comercio.

\subsubsection{Fase 1: actividades sobre el primer eje "habilidades técnicas".}

Los estudiantes realizarán actividades durante varios meses:

- Análisis de la situación del empleo en las áreas de los países participantes (mapa mental).

- Análisis de empresas mediante la elaboración de cuestionarios.

- Análisis de las habilidades técnicas de estudiantes y profesores (cuestionario para estudiantes y profesores). 
- Realización de cuestionarios, tablas, gráficos y presentación de resultados producidos por los estudiantes de cada país utilizando ICTsMeeting en Hoxter para la presentación de resultados.

\subsubsection{Fase 2: actividades sobre el segundo eje "habilidades sociales"}

- Reunión semanal con empresa-institución para el intercambio de información y recabar la misma.

- Del intercambio de los datos por parte surgirá un debate sobre la realidad empresarial y comercial de cada país, así como la realidad del mercado laboral. Esto ayudará a comprender el funcionamiento de cada país en estos términos.

- Creación de un decálogo de buenas prácticas para luchar contra el paro juvenil, compartiendo experiencias personales de los socios participantes.

- Crear una rúbrica para evaluar el decálogo del resto de los socios.

\subsubsection{Fase 3: "intercambio de prácticas"}

- Internacionalizar el enfoque de los estudios y de la futura práctica laboral de los alumnos a través de la experiencia internacional en empresas extranjeras.

- Fomentar en nosotros el intercambio de conocimientos teóricos y experiencias sobre el know how de la consultora extranjera. Así mismo, se pretende consolidar vínculos entre ambas entidades con la finalidad de crear nuevas relaciones colaborativas (centro integrado de formación profesional-empresa portuguesa).

Los alumnos serán registrados en el twinspace para facilitar la comunicación entre ellos.

\subsubsection{Fase 4: " difusión del proyecto"}

Una vez realizadas todas las fases anteriores cada grupo de trabajo realizará un póster donde se recoja todo lo trabajado en el proyecto y los resultados obtenidos:

- Difusión en las redes sociales del centro: Facebook, twitter y Twinspace.

- Posters informativos

Todo el proyecto se plantea en continua revisión. Además se contempla un plan de mejora del mismo, posibilitando la modificación de los acuerdos tomados con la intención de mejorar los resultados obtenidos.

\subsection{Herramientas}

- Twinspace: Los alumnos serán registrados en el twinspace para facilitar la comunicación entre ellos.

- My ebook: creación de libros interactivo a partir de un PDF.

- Voki.

- Móviles.

- PC.

- Youtube 


\subsection{Temporalización}

Tabla 2. Temporalización del Proyecto eTwinning

\begin{tabular}{ll}
\hline Julio & Jobshadowing en Lisboa (Portugal). \\
\hline Septiembre & Actividades sobre el primer eje "habilidades técnicas". \\
& $\begin{array}{l}\text { Mapa mental realizado por los alumnos sobre qué quieren trabajar en el proyecto, realización de } \\
\text { cuestionarios, tablas, gráficos y presentación de resultados producidos por los estudiantes de } \\
\text { cada país utilizando ICTsMeeting. }\end{array}$ \\
Octubre & $\begin{array}{l}\text { Presentación de los países participantes, ciudades,y estudiantes. Uso de la videoconferencia, } \\
\text { videos y Vokis para presentarse. }\end{array}$ \\
Noviembre & $\begin{array}{l}\text { Realización de un ebook entre todos los países con el decálogo de buenas prácticas para luchar } \\
\text { contra el desempleo juvenil. Con audio e imágenes. }\end{array}$ \\
Diciembre & $\begin{array}{l}\text { Elaboración y envío de CV (versión Europass) de los estudiantes que redactarán ellos mismos } \\
\text { en inglés. }\end{array}$ \\
Enero- & Entrevistas de trabajo online entre miembros de los diferentes países. \\
Febrero & Dar acceso al foro a los alumnos para que compartan sus análisis de situación, decálogo de \\
Marzo & buenas prácticas. \\
Mabril & Crear un padlet para que los alumnos compartan sus CV. \\
\hline
\end{tabular}

Fuente: Elaboración propia

\section{Conclusiones}

El reto que representa la materialización del presente proyecto se traduce en la necesidad de analizar y observar las actividades profesionales de empresas del exterior, cuya finalidad es la de estrechar vínculos entre ambas instituciones para aproximarnos al funcionamiento de la realidad empresarial internacional y, la manera de crear mecanismos que mejoren y hagan crecer los mercados internacionales, gracias a la reciprocidad entre el mercado portugués y el español. Así mismo, con ello se busca que el alumno se abra puertas al mercado laboral internacional.

A través de esta colaboración, se pretende conseguir una formación de calidad con la observación de métodos más innovadores que puedan ayudar al intercambio de buenas prácticas e ideas innovadoras. Todo ello, con el objetivo último de transmitir a nuestros alumnos la posibilidad futura de que sean ellos, quiénes tengan la oportunidad de trabajar en dicha empresa internacional.

Internacionalizar el enfoque de los estudios y de la futura práctica laboral de los alumnos a través de la experiencia internacional en empresas extranjeras. La posibilidad de realizar prácticas en el extranjero para nuestros alumnos, supone un valor añadido al CV de los 
mismos, aumenta su empleabilidad y mejora sus expectativas laborales, facilitándoles la transición del ámbito de la educación y la formación al mercado laboral. Las videoconferencias y actividades para facilitar la comunicación permiten que exista la interacción entre iguales y el uso de la lengua extranjera en un contexto real.

De este modo, mediante la introducción de metodologías innovadoras basadas en un aprendizaje colaborativo, se pretende que los alumnos aprendan de una manera diferente. Los objetivos de este proyecto facilitan la integración de diferentes áreas del currículo de los ciclos de educación superior.

\section{Referencias}

CEFIRE (2018). "Curso La dimensió europea a les aules: projectes eTwinning. Una perspectiva global (codi: 18TO75IN015)", (2018-06-10).

GALVIN, C. (2009). eTwinning in the classroom: A showcase of good practice (2008-2009). Brussels: Central Support Service for eTwinning \& European Schoolnet

INTEF (2018). "Curso en Red Proyecta eTwinning", Instituto Nacional de Tecnologías educativas y de formación del profesorado (FORMACIÓN EN RED) (2018-07-17).

INTEF (2017). "Curso Creación de Recursos Educativos Abiertos para la Enseñanza" Instituto Nacional de Tecnologías educativas y de formación del profesorado (FORMACIÓN EN RED) (201712-20).

MORENO, B. (2007). La dimensión europea de la Educación: Una investigación evaluativa en torno al programa eTwinning. Granada: Universidad de Granada.

PAZ-ALBO, J., LÓPEZ, I. (2017). Higher education perspectives on etwinning: the future of initial teacher training learning. 11th International Technology, Education and Development Conference. It will be published by IATED in the INTED2017 Proceedings, 6th-8th March 2017, Valencia, Spain.

Congreso mundial sobre los Recursos Educativos Abiertos (REA) UNESCO, París, 20-22 de junio de $2012 . \quad$ Disponible en: $<$ http://www.unesco.org/new/fileadmin/MULTIMEDIA/HQ/CI/CI/pdf/Events/Spanish_Paris_OER_ Declaration.pdf $>$ [Consulta: 10 de marzo de 2019].

Horizon Report. Resumen Informe Horizon 2015. Enseñanza Universitaria. Instituto de Tecnologías Educativas Departamento de Proyectos Europeos. Disponible en: <https://intef.es/wpcontent/uploads/2015/08/Resumen_Horizon_Universidad_2015_INTEF_agosto_2015.pdf $>$ [Consulta: 10 de marzo de 2019].

Portal oficial eTwinning. Disponible en: <https://etwinning.net $>$ [Consulta: 10 de marzo de 2019].

Portal oficial SEPIE. Disponible en: < http://sepie.es/> [Consulta: 10 de marzo de 2019]. 